\title{
Compact hybrid plasmonic polarization rotator
}

\author{
J. Niklas Caspers, ${ }^{*}$ M. Z. Alam, and Mo Mojahedi \\ Department of Electrical and Computer Engineering and Institute for Optical Sciences, \\ University of Toronto, 10 King's College Rd., Toronto, Ontario M5S 3G4, Canada \\ ${ }^{*}$ Corresponding author: n.caspers@utoronto.ca \\ Received July 20, 2012; revised August 14, 2012; accepted August 23, 2012; \\ posted August 24, 2012 (Doc. ID 173055); published November 6, 2012
}

We propose a novel ultracompact $(5 \mu \mathrm{m})$ hybrid plasmonic polarization rotator operating at telecommunication wavelength for integrated silicon photonic circuits. The polarization mode of a silicon waveguide is rotated with $>14 \mathrm{~dB}$ polarization extinction ratio and low total insertion losses of $2.1 \mathrm{~dB}$. (c) 2012 Optical Society of America OCIS codes: $130.3120,130.5440,230.3120,230.5440,240.6680$.

Integrated optical circuits if combined with fiber communication will require polarization diversity [1]. A polarization rotator will be a key component for such polarization diversity. However, it has been an ongoing challenge to design an integrated, compact low-loss rotator [2-4]. So far, most designs have been based on mode interference [2] or adiabatic mode evolution [3,4]. Both methods come with their own set of advantages and limitations: devices based on mode interference are short $(\sim 10 \mu \mathrm{m})$, but are sensitive to fabrication imperfections and tend to have a limited $3 \mathrm{~dB}$ bandwidth ( 25 nm) [2]. On the other hand, mode evolution schemes require rather long length $(>100 \mu \mathrm{m})$ to achieve sufficient extinction ratios and low insertion losses; additionally, they usually require uncommonly thick silicon waveguides [3] or additional material layers [4].

Recently, some authors have suggested the use of surface plasmon polaritons (SPPs) to reduce the length of a rotator [5,6]. For example, Zhang et al. demonstrated an ultracompact rotator $(3 \mu \mathrm{m})$ based on SPPs, but their design showed a high insertion loss $(>11 \mathrm{~dB})$ [5] .

Here, we propose using a hybrid plasmonic (HP) waveguide to design an ultra-compact integrated polarization rotator operating at $1.55 \mu \mathrm{m}$. The $\mathrm{HP}$ waveguide consists of a metal (e.g., silver) separated from a highindex medium (e.g., silicon) by a thin dielectric spacer (e.g., silica). The HP modes supported by the structure are the result of the coupling between the SPP modes existing at a metal/spacer interface and the modes supported by the high-index (silicon) waveguide. The HP waveguide was first reported in [7] as a compromise between the high confinement of the SPP waves and the low loss of the dielectric waveguide modes. Previously a TE-pass polarizer based on the HP structure has been reported [8]].

Our proposed HP polarization rotator combines the advantages of both mode evolution and mode interference schemes, while at the same time showing a low insertion loss $(\sim 2 \mathrm{~dB})$. The proposed polarizer can be made exceptionally short $(\sim 5 \mu \mathrm{m})$, while showing performances better or comparable to other state-of-the-art designs.

Figure 1 shows a schematic of the proposed polarization rotator. The rotator consists of three sections: the first section is a short taper that transforms the TM silicon waveguide mode into a HP mode. A silica spacer layer (omitted in Fig. 1 for clarity) separates the silver from the silicon throughout the device. A significant portion of the HP mode energy is located in this silica spacer.
The polarization rotation happens in the second section, where the silver layer moves sideways and downward relative to the silicon nanowire. Thus the silver rotates around the silicon waveguide. In the last section, the silver is terminated and the silicon waveguide linearly tapers out to its original width.

The input and output waveguide of the rotator is a standard silicon waveguide with a thickness of $220 \mathrm{~nm}$ and a width of $450 \mathrm{~nm}$. The silver layer has a thickness of $200 \mathrm{~nm}$ and a width of $300 \mathrm{~nm}$. The silver is located above and on the side of the silicon, separated from the silicon by a silica spacer layer. In our simulations, we have considered different spacer thicknesses between 50 and $110 \mathrm{~nm}$.

We propose a fabrication scheme that does not require a complicated three-dimensional (3D) etching profile, despite the silver rotating in a 3D fashion around the silicon waveguide. First, the silicon waveguide is etched into a standard silicon-on-insulator (SOI) wafer. Then the silica spacer layer is deposited using a thin film deposition technique (e.g., plasma-enhanced chemical vapor deposition). Last, resist is deposited and patterned to create a "window" for silver deposition. The window is directly
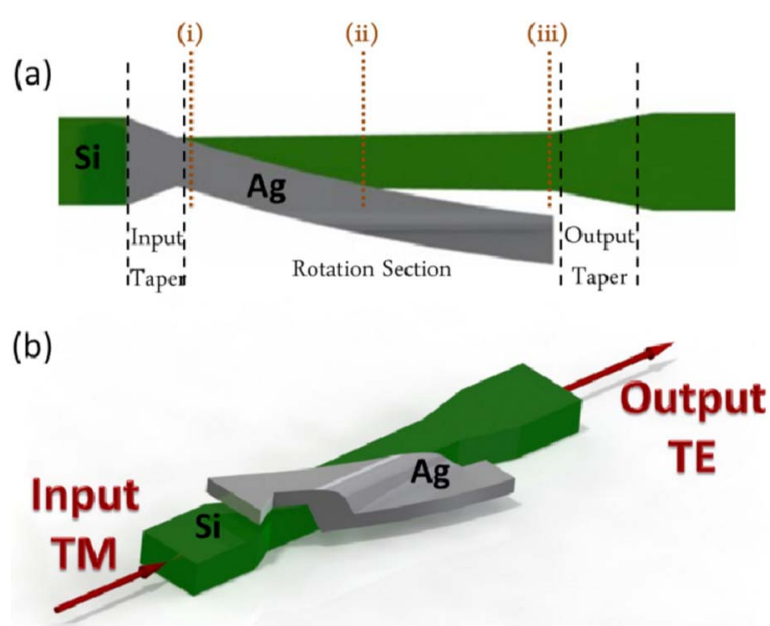

Fig. 1. (Color online) (a) Top view of the proposed polarization rotator, where the black dashed lines separate the three sections: input taper, rotation section, and output taper. The orange dotted lines indicate the cross sections for which the mode profile is plotted in Fig. 2(b). (b) 3D schematics (not to scale) of the proposed rotator. Green corresponds to silicon, grey is silver. The silica is not shown for clarity. 
above the silicon waveguide at the input waveguide/HP section interface and slowly turns away to the side in the longitudinal direction; it is completely located on the side of the waveguide at the end of the rotation section. Next, silver is deposited (e.g., thermal evaporation) and a liftoff is performed, resulting in a silver layer following the height change of the silica (due to the underlying silicon waveguide) and the pattern of the window. This will result in a silver layer as shown in Fig. 1 .

Despite silver not being a perfect conductor in the near infrared, it can be still considered a "good conductor" (due to its relative low losses) and thus the electric field will stay normal to the silver surface. The hybrid mode will rotate with the rotation of the silver layer around the silicon waveguide. The evolution of the TM hybrid mode at the input to a TE mode at the output is shown in Fig. 2. Here, we have plotted the norm of the electric field $(\|\vec{E}\|)$ of the HP mode for three positions along the rotator (positions are indicated in Fig. 1).

The final device was simulated using the 3D finite difference time domain (FDTD) solutions from Lumerical [9]. We properly choose the mesh density and simulation size to ensure a high accuracy. If otherwise not indicated, all simulations presented here are for a wavelength of $1.55 \mu \mathrm{m}$. For a suboptimal device design, the power in the output waveguide will not only be located in the wanted TE mode but some power might remain in the TM mode. Thus the power in the two polarizations needs to be separated. The power in the TM polarization was evaluated according to [10]

$$
P_{\mathrm{TM}}=\operatorname{Re}\left[\frac{\int\left(\vec{E}_{3 D} \times \vec{H}_{\mathrm{TM}}^{*}\right)_{z} \mathrm{~d} A \cdot \int\left(\vec{E}_{\mathrm{TM}} \times \vec{H}_{3 D}^{*}\right)_{z} \mathrm{~d} A}{\int\left(\vec{E}_{\mathrm{TM}} \times \vec{H}_{\mathrm{TM}}^{*}\right)_{z} \mathrm{~d} A}\right],
$$

where a similar expression was used for the TE polarization. Here, $\vec{E}_{3 D}$ and $\vec{H}_{3 D}$ are the electric and magnetic fields respectively extracted from the output waveguide of the 3D-FDTD device simulations. $\vec{E}_{\mathrm{TM}}$ and $\vec{H}_{\mathrm{TM}}$ are the fields of the TM mode of a silicon waveguide with the same dimensions $(220 \mathrm{~nm} \times 450 \mathrm{~nm})$ calculated using the FDTD mode solver from Lumerical. The integral is performed over a cross-sectional area of the output and $z$ refers to the propagation direction. The extracted powers in the respective polarizations were used to calculate the extinction ratio. The polarization extinction ratio is defined as the ratio of the transmitted powers in the TM and TE modes:

$$
\mathrm{ER}=-\log _{10}\left(\frac{P_{\mathrm{TM}}}{P_{\mathrm{TE}}}\right)
$$

The insertion loss (IL) was extracted, as well, where the power in the wanted waveguide mode (TE) is normalized to the input power. In all our simulations, the insertion loss is the total loss going from the silicon waveguide TM mode to the TE mode of the same Si waveguide. This includes the reflection loss to the hybrid mode at the interface to the input taper, both (input and output) taper losses, polarization mode conversion losses, and propagation losses due to the absorption in the metal. The input taper has a length of $200 \mathrm{~nm}$ and contributes $<0.3 \mathrm{~dB}$
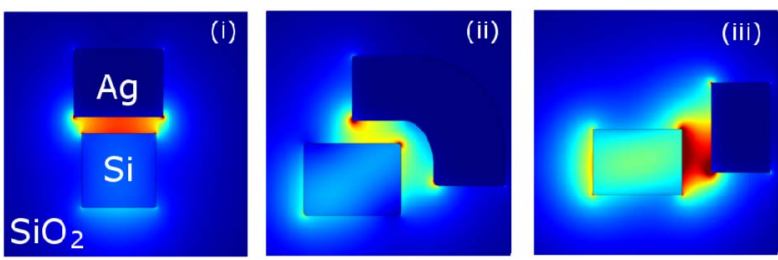

Fig. 2. (Color online) Norm of the electric field $(\|\vec{E}\|)$ profiles of the rotated mode along the waveguide. Positions of the profile along the rotator are indicated in Fig. 1(a). The positions correspond to (i) input, (ii) middle, and (iii) output of the rotation section.

loss to the total insertion loss. The output taper has a length of $1 \mu \mathrm{m}$ and has a similar loss of $<0.2 \mathrm{~dB}$.

We began optimizing the performance of the rotator by varying the width of the silicon nanowire in the rotation section [see Fig. 1(a)]. The results of the optimization are plotted in Fig. 3. For these simulations, the rotator had a total device length of $5.2 \mu \mathrm{m}$ and the silica spacer was $80 \mathrm{~nm}$ thick. The insertion loss is plotted in Fig. 3(a) as a function of the width of the silicon waveguide at the input of the rotation section [region marked (i) in Fig. 1(a)] and the width of the output [region marked (iii) in Fig. 1(a). A minimum insertion loss of $2.1 \mathrm{~dB}$ can be achieved for an input width of $220 \mathrm{~nm}$ and an output width of $300 \mathrm{~nm}$. A linear taper is used along the rotator section to match the two widths. The losses remain low for usual fabrication tolerances of $\pm 30 \mathrm{~nm}$, but rise significantly for larger deviations. The minimum loss coincides with a square cross section at the input.

(a)

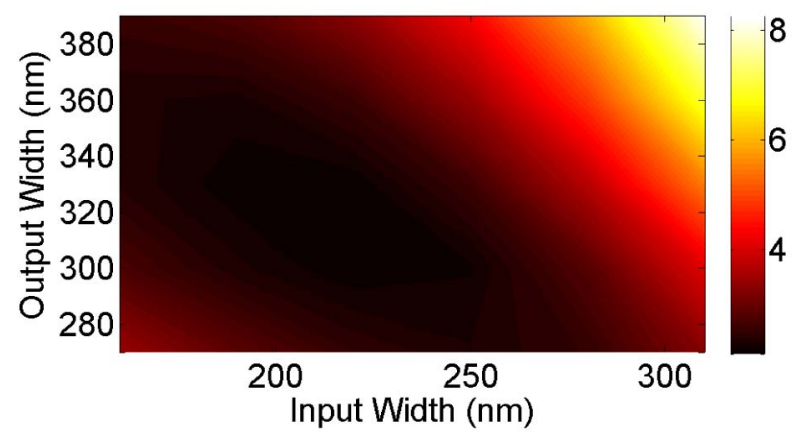

(b)

Extinction Ratio (dB)

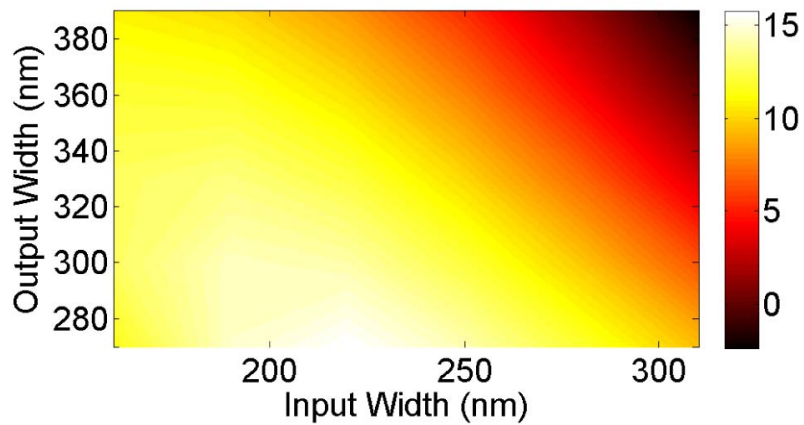

Fig. 3. (Color online) (a) Insertion loss and (b) the polarization extinction ratio between the TM and TE modes of the rotator as a function of the silicon-waveguide width at the input (horizontal axis) and output (vertical axis) of the rotation section. The graph is color coded, with brighter colors corresponding to higher values. 
Total Device Length $(\mu \mathrm{m})$

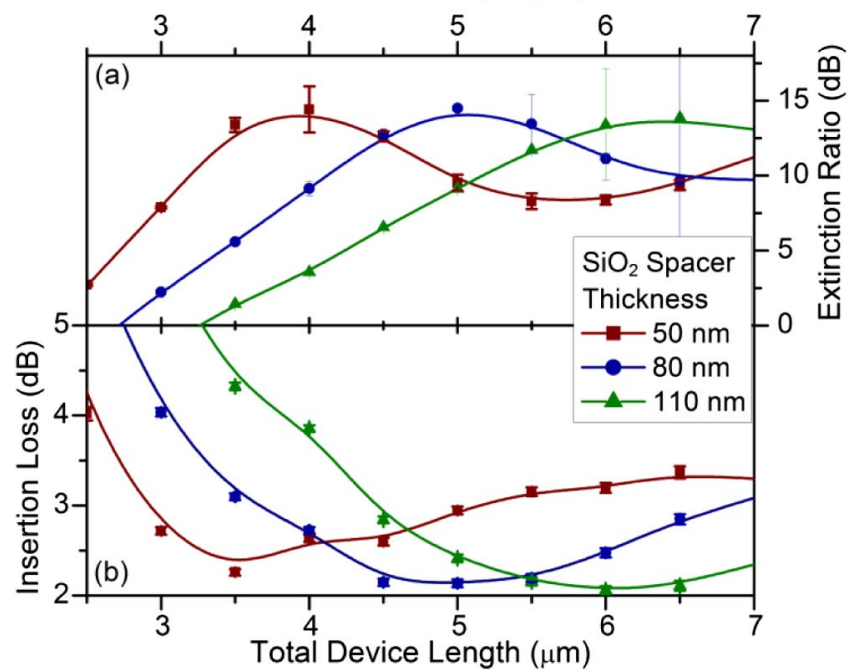

Fig. 4. (Color online) (a) Extinction ratio between the TM and TE modes for the proposed rotator as a function of device length, and (b) insertion loss for the same device. Symbols represent simulation results, while the curves are guides to the eye.

Figure 3(b) shows the extinction ratio again as a function of silicon waveguide widths. The highest extinction ratio is $15 \mathrm{~dB}$ for an input width of $220 \mathrm{~nm}$ and an output width of $270 \mathrm{~nm}$. While the optimal input width (220 nm) is the same for minimum insertion loss and maximum extinction ratio, the output width differs by $30 \mathrm{~nm}$. A wider cross section will increase the mode confinement in the silicon and, thus, the metal will be less effective in rotating the mode. However, for a too narrow silicon waveguide width, the insertion loss due to the metal, as well as losses due to the taper transitions, are expected to increase.

To further improve the device performance, we have simulated different device lengths and spacer thicknesses (Fig. 4), where the width of the silicon waveguide and the input and output of the rotation section was set to achieve the optimum insertion loss (input width $=220 \mathrm{~nm}$, output width $=300 \mathrm{~nm}$ ).

The extinction ratio as a function of device length is shown in Fig. 4(a). For a spacer thickness of $50 \mathrm{~nm}$, a device length of $4 \mu \mathrm{m}$ (the shortest device length) maximizes the extinction ratio, which is equal to $14.5 \mathrm{~dB}$ at its maximum. Figure 4(a) also indicates that, for thicker spacers and longer devices (for example, spacer thickness $=80$ or $110 \mathrm{~nm}$ and device lengths $=5$ or $6.5 \mu \mathrm{m}$ ), similar values for the extinction coefficient can be obtained. The extinction ratio remains high for devices longer than the maximum extinction ratio lengths, because the TM mode is still sufficiently suppressed.

The insertion loss as a function of device length for the same design is plotted in Fig. 4(b). The insertion loss drops to $2 \mathrm{~dB}$ for a silica thickness of $80 \mathrm{~nm}$ and a device length of $5 \mu \mathrm{m}$. Moreover, the minimum insertion loss is achieved for approximately the same device length that displays maximum extinction ratio. The insertion loss graph shows the two loss regimes. (I) If the rotator length is too short $(<4 \mu \mathrm{m})$, the mode is not adiabatically rotated and, thus, power is coupled and lost to other modes. (II) If the rotator is too long $(>7 \mu \mathrm{m})$, it will suffer from material losses due to the silver. The optimal device length depends on the silica spacer thickness, but is approximately between 4 and $7 \mu \mathrm{m}$. The $3 \mathrm{~dB}$ bandwidth of the extinction ratio is $>150 \mathrm{~nm}$, thus the design shows a broadband characteristic as expected for a mode evolution scheme.

In summary, an ultracompact HP polarization rotator for integrated optical silicon photonic circuits was designed. The total device length can be as short as $5 \mu \mathrm{m}$, while reaching an extinction ratio $>14 \mathrm{~dB}$ and showing a low insertion loss of $2.1 \mathrm{~dB}$. The extinction ratio could be further improved by using the rotator in tandem with an integrated polarizer similar to the one reported in [8]. By using HP waveguides, more compact and efficient devices with various functionalities for integrated optical circuits, on a silicon compatible platform, can be realized.

\section{References}

1. M. Lipson, J. Lightwave Technol. 23, 4222 (2005).

2. A. V. Velasco, M. L. Calvo, P. Cheben, A. Ortega-Moñux, J. H. Schmid, C. A. Ramos, Í. M. Fernandez, J. Lapointe, M. Vachon, S. Janz, and D.-X. Xu, Opt. Lett. 37, 365 (2012).

3. J. Zhang, M. Yu, G.-Q. Lo, and D.-L. Kwong, IEEE J. Sel. Top. Quantum Electron. 16, 53 (2010).

4. L. Chen, C. Doerr, and Y.-K. Chen, Opt. Lett. 36, 469 (2011).

5. J. Zhang, S. Zhu, H. Zhang, S. Chen, G.-Q. Lo, and D.-L. Kwong, IEEE Photon. Technol. Lett, 23, 1606 (2011).

6. M.-a. Komatsu, K. Saitoh, and M. Koshiba, IEEE Photon. J. 4, 707 (2012).

7. M. Z. Alam, J. Meier, J. S. Aitchison, and M. Mojahedi, in Conference on Lasers and Electro-Optics/Quantum Electronics and Laser Science Conference and Photonic Applications Systems Technologies, OSA Technical Digest (CD) (Optical Society of America, 2007), paper JThD112.

8. M. Z. Alam, J. S. Aitchison, and M. Mojahedi, Opt. Lett. 37, 55 (2012).

9. www.lumerical.com.

10. A. Yariv and P. Yeh, Photonics-Optical Electronics in Modern Communications (Oxford, 2007). 